\title{
Loss allocation in distribution networks with distributed generators undergoing network reconfiguration
}

\author{
Ambika Prasad Hota1, Sivkumar Mishra ${ }^{2}$ \\ ${ }^{1}$ International Institute of Information Technology Bhubaneswar, India \\ ${ }^{2}$ Centre for Advanced Post Graduate Studies, Biju Patnaik University of Technology, India
}

\begin{tabular}{l}
\hline Article Info \\
\hline Article history: \\
Received Apr 3, 2019 \\
Revised Jan 13, 2020 \\
Accepted Jan 30, 2020 \\
\hline
\end{tabular}

Keywords:

Distributed generator

Loss allocation

Network reconfiguration

Radial distribution network

\begin{abstract}
In this paper, a branch exchange based heuristic network reconfiguration method is proposed for obtaining an optimal network in a deregulated power system. A unique bus identification scheme is employed which makes the load flow and loss calculation faster due to its reduced search time under varying network topological environment. The proposed power loss allocation technique eliminates the effect of cross-term analytically from the loss formulation without any assumptions and approximations. The effectiveness of the proposed reconfiguration and loss allocation methods are investigated by comparing the results obtained by the present approach with that of the existing "Quadratic method" using a 33-bus radial distribution system with/without DGs.
\end{abstract}

Copyright $\odot 2020$ Institute of Advanced Engineering and Science. All rights reserved.

Corresponding Author:

Ambika Prasad Hota,

International Institute of Information Technology Bhubaneswar,

Bhubaneswar, Odisha, India.

Email: c117001@iiit-bh.ac.in

\section{INTRODUCTION}

In a deregulated environment and with the emergence of smart grids, it is the consumers and distributed generators (DGs) of the distribution system (DS), who will ultimately pay or get paid for their individual share of network utilization. Active power loss in electricity distribution is a prominent component of this network utilization cost. Hence, it is to be recovered from the network users by suitably and fairly allocating it among them. The nonlinear relationship between power loss and injected powers makes loss allocation (LA) process difficult and complicated [1]. Simultaneously, in electric power distribution networks (EPDNs), there is a growing trend in the penetration of DGs because optimal placement of DGs in the EPDNs often lead to substantial reduction in active power loss [2]. Therefore, it is essential to allocate this decrease in power loss among the network participants judiciously as per their actual contribution towards system loss reduction (SLR). Further, LA to the end users get affected due to the changing network topology i.e. network reconfiguration (NR), which is also an established method of loss reduction in EPDNs. Thus, based on one's changing relative position in the network and the type of load, a consumer/DGO may either have to get rewarded or penalized. Hence, there is a scope to analyze the impact of NR on active power loss allocation in modern distribution networks, particularly, when very little work has been reported where both NR and LA are considered together.

Literature survey reveals that most of the LA methods were initially proposed for transmission systems [3], and later, LA methods exclusively meant for distribution systems are reported in Refs [4-11]. With the penetration of DGs, the LA methods are modified and proposed accordingly. A power summation based LA algorithm is developed in [5] where the cross-term of power loss equation is bifurcated among the responsible nodes using a quadratic scheme. This technique is further extended for energy calculation in Ref. [6] through a statistical analysis of daily load and generation curves of DGs. In [7], a three stepped LA 
procedure is discussed where in the first step; losses are assigned to loads then in the next step to DGs. In the third step normalization technique is used for final settlement of the losses. However, the method discussed in [8] does not require any reconciliation procedure for allocation of losses where the cross-terms are shared using a geometric scheme of LA. Kashyap and De [9] have proposed a proportional sharing based LA scheme for distribution of losses among the consumers and DG units. In this approach, loads are assigned losses according to their contribution and then DGs are allocated by considering a contribution matrix followed by power sharing matrix. The method developed in [10] uses a participation matrix to identify the participation of nodes in the loss of each branch. An analytical cooperative game theory based technique is followed in the developed method [12] with Shapley value to overcome computational burden along with fair allocation. Kumar et al. [13] developed a branch-oriented circuit theory based LA technique which uses a contribution based loss allocation factor for bifurcation of the cross-terms present in the power loss equation.

Moreover, Mishra et al. [14] presented a comprehensive review of various NR methods up to 2016. Heuristic-based NR methods converge very quickly as compared to the meta-heuristic search based NR methods [15]. However, heuristic methods do not always guarantee a global minimum configuration. In [16], a branch exchange based heuristic NR algorithm is proposed for the fulfillment of reliability and power quality based objectives. Ghasemi [17] suggested a heuristic NR algorithm for reliability based indices. Gamapa and Das [18] used a heuristic method for a fuzzy multi-objective NR with DGs. Das et al. [19] proposed a heuristic, circular mechanism for NR in the presence of DGs. In [20], a two-stage heuristic NR method using optimal load flow is introduced. Tyagi et al. [21] also proposed a two-stage heuristic NR algorithm for loadability enhancement. Jasthi and Das [22] proposed a loss formula based heuristic method for NR. As NR is proven to be the most effective means of minimizing active power loss for EPDNs, so, it is essential to study the impact of NR on the loss allocation methods in a distribution system, where it is assumed that the consumers have to pay for the losses or sometimes get rewarded. Oliveira et al. [23] were the first to consider NR and LA together for distribution systems with DGs, where the NR is executed using a heuristic method and the loss allocation using Z-bus method [24], but the findings were not very convincing. Savier and Das [25] studied the impact of NR on LA in a much better way, where a heuristic branch exchange based algorithm is employed for optimal reconfiguration, and then quadratic loss allocation scheme is used to allocate losses to various consumers in a radial distribution network (RDN) before and after the reconfiguration. Recently, a metaheuristic based firefly algorithm [26], a modified FBS based technique [27] and a Grey Wolf optimization method [28] are also introduced for obtaining an efficient reconfigured power distribution network with improved voltage profile.

In the light of the above developments, this paper carries the analysis further. For fair allocation, a new loss allocation technique is employed in section-2. Simultaneously, system loss is reduced using a heuristic branch exchange (BE) based NR approach, which is described in section-3. As the LA scheme uses the converged load flow results, and in NR, successive load flows are performed for changing network topologies with successive branch exchanges, a forward-backward sweep based load flow technique as described in [2] is implemented with a unique bus identification scheme. In section-4, the efficiency of the proposed method is evaluated by comparing the LA results with the existing Quadratic method at both before and after NR considering a 33-bus RDN with/without DGs. Conclusive remarks are made in section-5.

\section{NEW METHOD OF LOSS ALLOCATION}

The proposed LA scheme is explained by considering a sample 10-bus RDN as shown in Figure 1. For the sake of simplicity in the analysis, the substation bus is indexed as ' 1 ' and the subsequent buses along the main feeder and lateral feeders are indexed in the increasing order. The branch number is one unit less than that of its receiving end node number. In this type of RDN, the total number of branches (nbr) is one unit less than that of the total number of nodes $(\mathrm{nb})$ in the network.

\subsection{Proposed bus identification scheme}

To identify the adjacent buses and the subsequent buses in a RDN, several arrays are proposed. These arrays make the load flow faster due to its reduced search time. The adjacent buses in the RDN are detected and saved in an array $\mathrm{adb}[]$ of dimension twice the nbr. Two pointer arrays of dimension equal to the number of buses ' $\mathrm{nb}$ ' are presented, where the arrays $\mathrm{mf}$ [i] and $\mathrm{mt}[\mathrm{i}]$ are used to point the starting and ending memory locations in the adb[] array, respectively. Similarly, an array pb[] is used to identify and store all the previous buses of the RDN. The constructions of these arrays are performed using network data and simple programming techniques. The adjacent and previous bus data of the 10-bus RDN as shown in Figure 1 are stored in the arrays $\mathrm{mf}, \mathrm{mt}$, adb and $\mathrm{pb}$ as shown in Figure 2 to describe the storage and pointer operation of these arrays. 
Similarly, two other arrays $\mathrm{nsb}[]$ and $\mathrm{sb}[]$ are proposed for providing information about the subsequent buses. All the subsequent buses corresponding to each branch of the RDN are stored in the array $\mathrm{sb}[]$, and their respective numbers (i.e. the number of subsequent buses) are stored in the array nsb[] (dimension equal to nbr). The starting and ending memory locations of the $s b[]$ array are pointed out by two pointer arrays $\mathrm{mfs}$ and $\mathrm{mts}$, respectively. The subsequent bus data of the 10-bus RDN as shown in Figure 1 along with the representation of branch-(2) are specified in the arrays sb, nsb, mts and mfs as shown in Figure 3. These arrays are formed using the network data and simple programming techniques in MATLAB (R2018b) environment. The utilization of the proposed arrays in both load flow (LF) analysis and loss allocation reduce the computational time and hence, works more effectively for larger RDNs. In each stage of $\mathrm{NR}$, the arrays are reconstructed based on the reconfigured RDN and system loss calculation is performed for obtaining optimal reconfiguration.

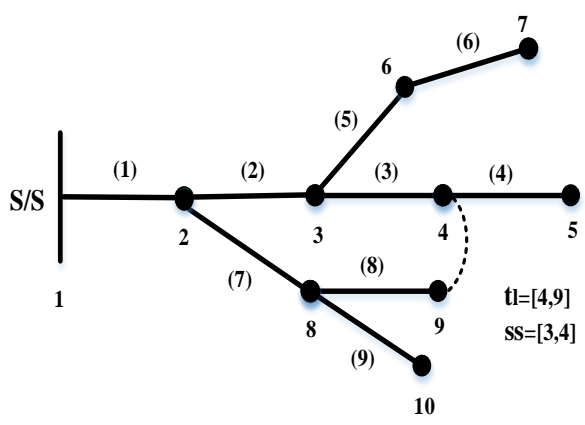

Figure 1. A Sample 10-Bus RDN

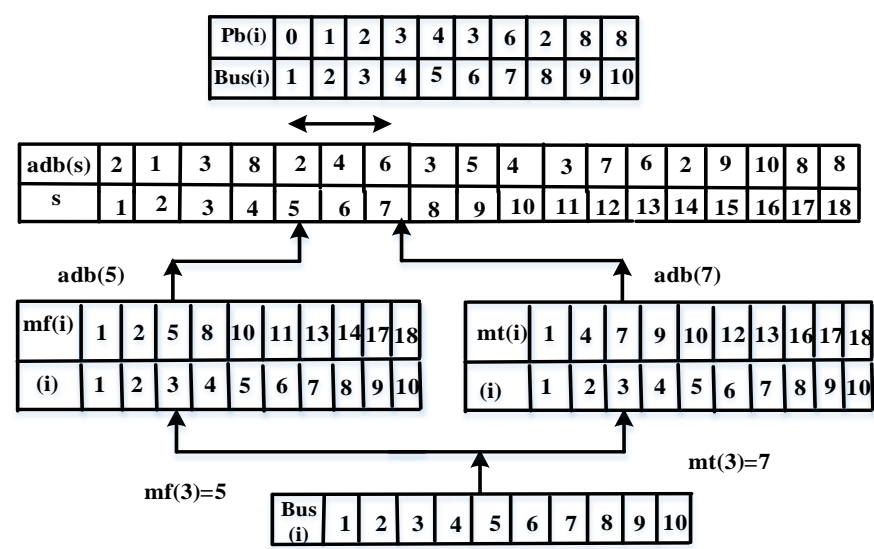

Figure 2. Storage and pointer operations of $\mathrm{mf}, \mathrm{mt}, \mathrm{pb}$ and adb arrays

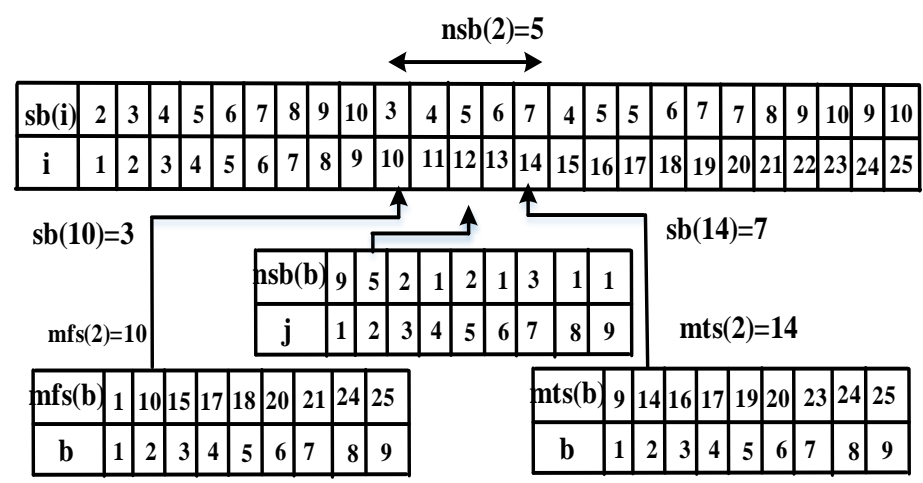

Figure 3. Storage and pointer operations of $\mathrm{mfs}$, mts, $\mathrm{sb}$ and nsb arrays 


\subsection{Formulation of the LA method}

In this paper, a forward backward sweep (FBS) based load flow method as discussed in Ref. [2] is carried out for obtaining the converged values of bus voltages as these are the prerequisites for power loss allocation. Further, the injection of DG power affects power loss of a system. Hence, in order to include DGs into the computational process the negative load modeling of DGs [8] is followed. Thus, considering DGs as negative loads, the equivalent current injection (ECI) at any arbitrary bus-i of the RDN with net node power injection "SLi = PLi + jQLi" and bus voltage "Vi" can be computed as:

$$
I_{L i}=\left[\frac{\left(P_{L i}+j Q_{L i}\right)}{V_{i}}\right]^{*}=\frac{\left(P_{L i}-j Q_{L i}\right)}{V_{i}^{*}}, \text { for } i=2,3, \ldots \ldots ., n b
$$

The current of branch- $b$ can be calculated by adding the ECIs of the subsequent buses with the help of $s b, m t s$ and $m f s$ arrays as:

$$
I(b)=\sum_{i=m f s(b)}^{m t s(b)} I_{L s b(i)}
$$

Here, $I_{L s b(i)}$ represents the ECI of the subsequent consumer of branch- $b$ which is connected at node- $i$ The branch current can be expressed in terms of complex power of the subsequent load points as:

$$
I(b)=\sum_{i=m f s(b)}^{m t s(b)} \frac{P_{L s b(i)}-j Q_{L s b(i)}}{V_{s b(i)}^{*}}
$$

The real or active power loss of the branch- $b$ can be evaluated in terms of sending end voltage $\left(V_{s}\right)$, receiving end voltage $\left(V_{r}\right)$ and the branch current $I(b)$ as:

$$
P \operatorname{Loss}(b)=\operatorname{Re} a l\left[\left(V_{s}-V_{r}\right)^{*} I(b)\right]
$$

Where, '*' represents the conjugate value of a complex number.

Substituting (3) in (4)

$$
P \operatorname{Loss}(b)=\operatorname{Re} a l\left[\left(V_{s}-V_{r}\right)^{*} \sum_{i=m f_{s}(b)}^{m t s(b)} \frac{P_{L s b(i)}-j Q_{L s b(i)}}{V_{s b(i)}^{*}}\right]
$$

Rearranging,

$$
\begin{aligned}
& \operatorname{PLoss}(b)=\operatorname{Re} a l\left[\sum_{i=m f s(b)}^{m t s(b)}\left(\frac{V_{s}-V_{r}}{V_{s b}(i)}\right)^{*} \cdot\left(P_{L s b(i)}-j Q_{L s b(i)}\right)\right] \\
& \text { Let, }\left(\frac{V_{s}-V_{r}}{V_{s b(i)}}\right)^{*}=A_{s b(i)}+j B_{s b(i)}
\end{aligned}
$$

The expression of Real power loss for the branch- $b$ can be written as:

$$
P \operatorname{Loss}(b)=\sum_{i=m f s(b)}^{m t s(b)}\left\{A_{s b(i)} \cdot P_{L s b(i)}+B_{s b(i)} \cdot Q_{L s b(i)}\right\}
$$

It is observed from (8) that, the customers beyond branch- $b$ of the RDN are responsible for the active or true power loss of the branch- $b$. Hence, this loss can be allocated to the consumers those are connected to the subsequent buses of the branch- $b$ (i.e. all $s b(i)$, where $i=m f s(b)$ to $m t s(b)$ ). Therefore, the contribution of nodes to branch power loss can be computed as: 


$$
\operatorname{ploss}(b, s b(i))=\left\{A_{s b(i)} \cdot P_{L s b(i)}+B_{s b(i)} \cdot Q_{L s b(i)}\right\}
$$

Thus, the total loss allocation for a consumer at $i^{\text {th }}$ bus can be calculated as the addition of the corresponding loss allocations from each of the branch- $b$ of the RDN, and is represented in (10).

$$
\operatorname{Tploss}(i)=\sum_{b=1}^{n b r} \operatorname{ploss}(b, s b(i)) \quad \text { where } i=2,3, \ldots . n b
$$

Hence, the total real power loss of the system can be calculated as:

$$
\text { TPLoss }=\sum_{i=1}^{n b} \text { Tploss }(i)
$$

\section{PROPOSED HEURISTIC NETWORK RECONFIGURATION (NR) METHOD}

In the proposed heuristic NR method, an optimal RDN is obtained after a series of branch exchanges (BEs). Two heuristic rules are followed in the proposed BE technique to achieve a minimum loss RDN. The first rule decides the tie line (i.e. the first member of the BE pair) to be closed for forming a weakly meshed network. The radial nature of the network is regained by opening a selected branch (sectionalizing switch) from the loop so formed by using the second heuristic rule. The proposed BE algorithm is developed to maintain the radial structure of the RDN after each BE so that load flow can be performed effectively. Thus, the arrays Figures 2 and 3 are to be modified according to new RDN after each BE step for LF and loss calculation. The NR method is explained in detail in algorithm-1. In this paper, the normally open switch or tie line is denoted as 'tl', and the normally closed switch or sectionalizing switch is denoted as 'ss'.

Algorithm-1:

STEP-1: Read the input data of the RDN and assign, Ntl = Number of tie line in the RDN.

STEP-2: Assign $\mathrm{j}=1$

STEP-3: Execute load flow and calculate the real power loss (TPLoss) of the base network by the proposed method.

STEP-4: Find the potential difference across all the tie lines (i.e. VDi, for $\mathrm{i}=\mathrm{j}, \ldots . . \mathrm{Ntl}$ ) and identify the tie line with maximum potential difference (VDmax). Also, find the node voltages ( $\mathrm{Vp}$ and $\mathrm{Vq})$ of the tie line $(\mathrm{tl}=[\mathrm{p}, \mathrm{q}])$ having maximum potential difference VDmax, Let say $\mathrm{Vp}<\mathrm{Vq}$.

STEP-5: Close the tie line (tl) having maximum voltage difference (VDmax) and then open the branch or sectionalizing switch (ss) adjacent to the node-p of the (tl) to maintain radial structure of the network.

STEP-6: Compute the power loss (TPLoss-new) of the newly formed RDN by the proposed method using modified arrays.

STEP-7: Check TPLoss > TPLoss-new, if yes step-8 is executed else step-11 is followed.

STEP-8: Assign TPLoss = TPLoss-new

STEP-9: Check for all branches of the loop, if TPLoss > TPLoss-new, step-11 is executed otherwise step 10 is followed.

STEP-10: Select the branch adjacent to the previous branch as sectionalising switch for the same tie line (tl), and follow step- 6 .

STEP-11: Check, if $j=N t l$ proceed to step-12 else increase $\mathrm{j}$ by one unit and execute step- 4 .

STEP-12: The network obtained is the optimum configured network and the corresponding TPLoss is the minimum loss of the network.

\section{RESULTS AND DISCUSSION}

The proposed BE based heuristic NR techniques along with present LA scheme are implemented on a $12.66 \mathrm{kV}, 100 \mathrm{kVA}, 33$-bus RDN as shown in Figure 4 [4] in MATLAB (R2018b) environment for evaluating effectiveness of the developed LA procedures against the established Quadratic Scheme [25] with/without DGs. The related line and load data are collected from Ref. [4] and positions of DGs are identified at the buses 14, 18 and 32 based on the loss sensitivity analysis as discussed in [29]. The DGs at these buses inject active powers into the system i.e. $589.7 \mathrm{~kW}$ at bus- $14,189.5 \mathrm{~kW}$ at bus-18 and $1014.6 \mathrm{~kW}$ at bus-32. A new RDN is obtained in each BE step hence, the calculation of power loss is performed with the newly obtained RDN at each step of NR and compared with the previous value by following the LA

Loss allocation in distribution networks with distributed generators undergoing... (Ambika Prasad Hota) 
procedure as discussed in algorithm-1 in order to achieve an optimal RDN. The detail tie line data of the base and optimised 33-bus RDN with/without DG are presented in Table 1. The total real power loss of the base network is found to be $202.67 \mathrm{~kW}$ in the absence of DG units, and it gets reduced to $145.96 \mathrm{~kW}$ for the reconfigured RDN as obtained using the proposed BE based NR technique. Moreover, it can be noticed from the LA comparison Table 2, the RDN loss gets reduced to $88.67 \mathrm{~kW}$ at base case due to injection of DG power into the system. The implementation of the proposed NR technique again decreases this loss to $70.2 \mathrm{~kW}$ due to improvement in voltage profile of maximum load points. The voltage magnitudes of the network before and after NR in the presence/absence of DGs are computed and the corresponding graph is plotted in Figure 5.

It can be observed from this figure that both NR and DG power injection have significant impact on the improvement of voltage profile and consequently, on system loss reduction. Thus, the effect of PDNR may be realized from the improvement in voltage profiles at bus-18 and bus-33. Furthermore, the variation of voltages from substation bus to end buses are more in the base RDN without DGs, which can be considered as an unhealthy situation for a RDN. Also, it can be noticed, the fluctuations in voltages decrease in the reconfigured network.

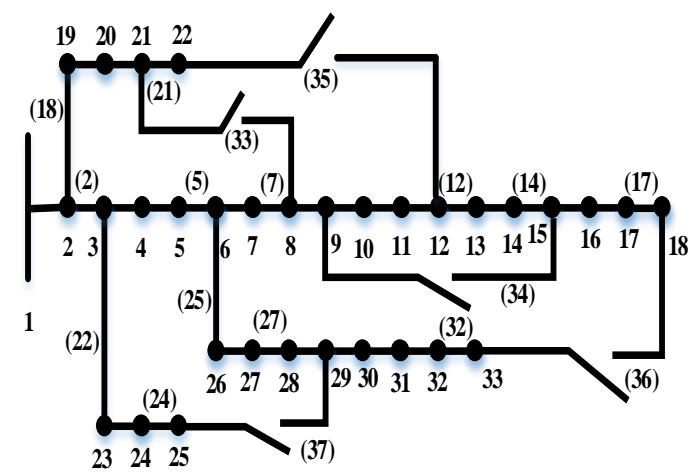

Figure 4. 33-Bus test System before NR without DGs

\begin{tabular}{lll} 
Table 1. Detail reconfiguration data of the considered 33-bus RDN with/without DGs \\
\hline 1 & Tie-line data of the base 33-bus RDN before NR without DGs & $33,34,35,36,37$ \\
2 & Total active power loss of the base 33-bus RDN before NR without DGs & $202.67 \mathrm{~kW}$ \\
3 & Tie-line data of the base 33-bus RDN after NR without DGs & $8,14,28,32,33$ \\
4 & Total active power loss of the base 33-bus RDN after NR without DGs & $145.96 \mathrm{~kW}$ \\
5 & Tie-line data of the base 33-bus RDN before NR with DGs & $33,34,35,36,37$ \\
6 & Total active power loss of the base 33-bus RDN before NR with DGs & $88.67 \mathrm{~kW}$ \\
7 & Tie-line data of the base 33-bus RDN after NR with DGs & $7,28,32,34,35$ \\
8 & Total active power loss of the base 33-bus RDN after NR with DGs & $70.2 \mathrm{~kW}$
\end{tabular}

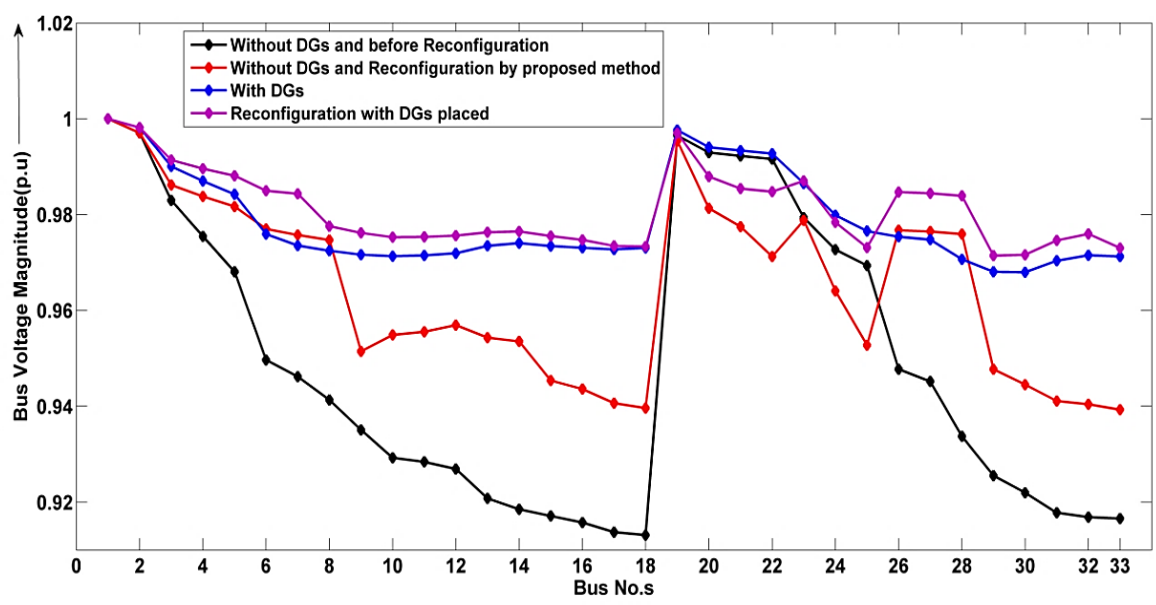

Figure 5. Voltage Profile of 33-Bus test system with/without DGs before and after NR 
Table 2. Loss allocation results of the 33-bus test system with/without DGs

\begin{tabular}{|c|c|c|c|c|c|c|c|c|}
\hline \multirow{3}{*}{$\begin{array}{l}\text { Bus } \\
\text { No. }\end{array}$} & \multicolumn{4}{|c|}{ Loss Allocation Results without DGs (in kW) } & \multicolumn{4}{|c|}{ Loss Allocation Results with DGs (in kW) } \\
\hline & \multicolumn{2}{|c|}{ Proposed LA scheme } & \multicolumn{2}{|c|}{ Quadratic scheme } & \multicolumn{2}{|c|}{ Proposed LA scheme } & \multicolumn{2}{|c|}{ Quadratic scheme } \\
\hline & Before NR & After NR & Before NR & After NR & Before NR & After NR & Before NR & After NR \\
\hline 1 & 0.3129 & 0.3089 & 0.2164 & 0.2158 & 0.2314 & 0.2301 & 0.1883 & 0.1888 \\
\hline 2 & 1.6303 & 1.3308 & 0.8743 & 0.5559 & 1.1042 & 0.9356 & 0.7277 & 0.4837 \\
\hline 3 & 3.2501 & 2.1136 & 2.71 & 1.4956 & 2.2395 & 1.5663 & 2.2047 & 1.2811 \\
\hline 4 & 2.1026 & 1.1679 & 0.8323 & 0.379 & 1.3079 & 0.8335 & 0.6941 & 0.3396 \\
\hline 7 & 12.3531 & 5.089 & 14.3433 & 5.3817 & 7.6204 & 5.2785 & 9.9153 & 8.4222 \\
\hline 8 & 4.1169 & 2.7291 & 1.7627 & 1.9175 & 2.19 & 1.6537 & 1.3135 & 1.3229 \\
\hline 9 & 4.4931 & 2.5241 & 2.0444 & 1.7362 & 2.2638 & 1.7653 & 1.4777 & 1.5147 \\
\hline 10 & 3.3633 & 1.632 & 1.4354 & 1.1777 & 2.0861 & 1.5216 & 1.1077 & 1.1594 \\
\hline 11 & 4.6141 & 2.1583 & 2.5991 & 1.9317 & 2.6507 & 1.977 & 1.9143 & 1.9925 \\
\hline 12 & 4.9821 & 2.3174 & 2.9232 & 2.0289 & 2.7044 & 2.0864 & 2.0599 & 2.1888 \\
\hline 17 & 8.1816 & 4.8948 & 6.6916 & 5.7436 & -1.259 & -1.8128 & -2.655 & -2.9087 \\
\hline 18 & 0.3183 & 0.406 & 0.1976 & 0.2996 & 0.2376 & 0.3054 & 0.1765 & 0.257 \\
\hline 19 & 0.5966 & 1.5323 & 0.476 & 1.5483 & 0.5151 & 1.2063 & 0.4541 & 1.2876 \\
\hline 20 & 0.6472 & 1.8186 & 0.527 & 1.8734 & 0.5656 & 1.4534 & 0.505 & 1.5483 \\
\hline 21 & 0.6911 & 2.2726 & 0.5714 & 2.3996 & 0.6094 & 1.4985 & 0.5492 & 1.5944 \\
\hline 22 & 1.9554 & 2.0651 & 1.0112 & 0.7699 & 1.4565 & 1.5015 & 0.8583 & 0.6645 \\
\hline 23 & 11.711 & 16.176 & 15.0305 & 18.013 & 9.2212 & 11.553 & 11.9454 & 13.087 \\
\hline 24 & 13.0342 & 21.583 & 16.3952 & 23.327 & 10.5186 & 15.033 & 13.2742 & 16.2481 \\
\hline 25 & 3.3886 & 1.4331 & 1.2892 & 0.4904 & 2.0026 & 0.9998 & 1.0298 & 0.4373 \\
\hline 26 & 3.5863 & 1.4487 & 1.3421 & 0.5064 & 2.0881 & 1.0151 & 1.0689 & 0.453 \\
\hline 27 & 4.3744 & 1.4765 & 1.4385 & 0.5068 & 2.3848 & 1.0295 & 1.118 & 0.4553 \\
\hline 32 & 5.7481 & 2.9926 & 2.2209 & 3.1558 & 3.5864 & 2.5359 & 1.6953 & 2.6865 \\
\hline Total LA $\rightarrow$ & 202.67 & 145.96 & 202.67 & 145.96 & 88.67 & 70.2 & 88.67 & 70.2 \\
\hline
\end{tabular}

Moreover, it can be observed from Table 2, the consumers connected from bus-2 to bus-11 are having a fair allocation after reconfiguration as regard to their respective load values and geographical locations. All the customers in this group get benefited due to NR by the proposed LA approach. But, the customer at bus 9 is allocated with more loss by Quadratic method, which is unfair. Customers at bus-19 to 22 get higher allocations by both the LA methods because, the relative locations of these consumers changes due to reconfiguration which leads to decrease in the voltage magnitudes for these customers. Due to the same above reason, the customers at bus- 23 to 25 get higher allocations. But, the proposed LA technique always allocates lesser losses to heavily loaded customers as compared to Quadratic LA method.

The effectiveness of the proposed method during the simultaneous impact of DG and NR can be evaluated from the LA data of Table 2. The loss allocation of the DG connected consumer at bus -14 is first analysed. The relative location of this consumer is not affected by the NR. The proposed method assigns almost an equal amount of loss $(-9.55 \mathrm{~kW})$ at both the conditions of the network whereas, Quadratic scheme allocates less benefit $(-11.98 \mathrm{~kW})$ to the load point after NR as compared to before NR $(-15.52 \mathrm{~kW})$, which is undesired. The DG connected consumers at buses-18 and 32 have benefitted because of NR by both the LA methods. However, the present approach provides an adequate amount of incentives to the DG connected nodes 18 and 32 after NR against Quadratic method. It is identified that the consumers at bus-19 to 25 and 32 have higher LA after NR by both the methods. But, all remaining consumers are allocated with higher losses after reconfiguration by Quadratic LA while the proposed method allocates lesser losses.

Further, in order to analyse response of the RDN as regard to geographical locations, two sets of consumers with equal load demands are identified. The first set consists of two consumers situated far away from each other i.e. at nodes 6 and 28. Both methods are allocating more loss to consumer at bus 28 than that of at node 6 but, the discrimination between their LA is more prominent in the present procedure which can be verified from Figure 6. Similarly, the discrimination between two close customers connected at node 16 and 17 as shown in Figure 7 is better in the proposed scheme as compared to Quadratic method at before/after reconfiguration of the network with/without DGs. At both scenarios, the developed method is providing promising results against Quadratic method. Hence, the present approach of loss allocation is found suitable to be implemented in the practical field of application for fair allocation with optimal network reconfiguration.

Loss allocation in distribution networks with distributed generators undergoing... (Ambika Prasad Hota) 


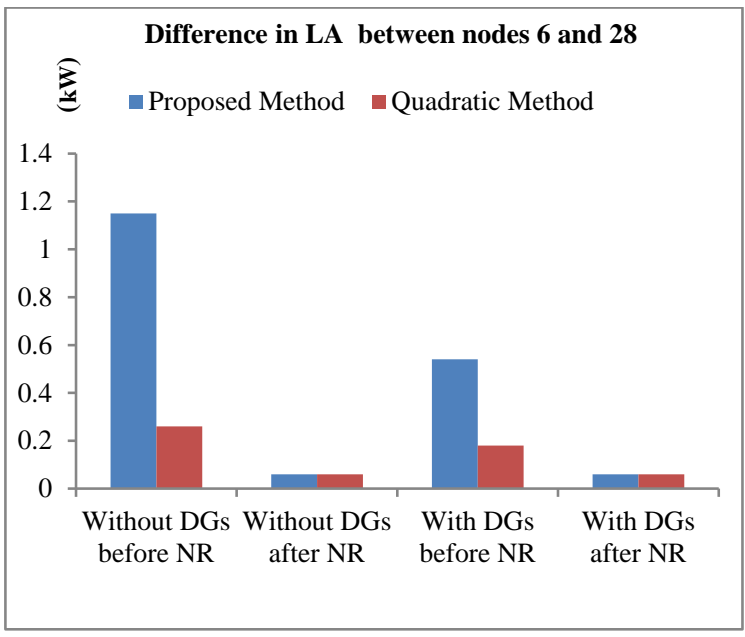

Figure 6. Difference in LA between nodes 6 and 28

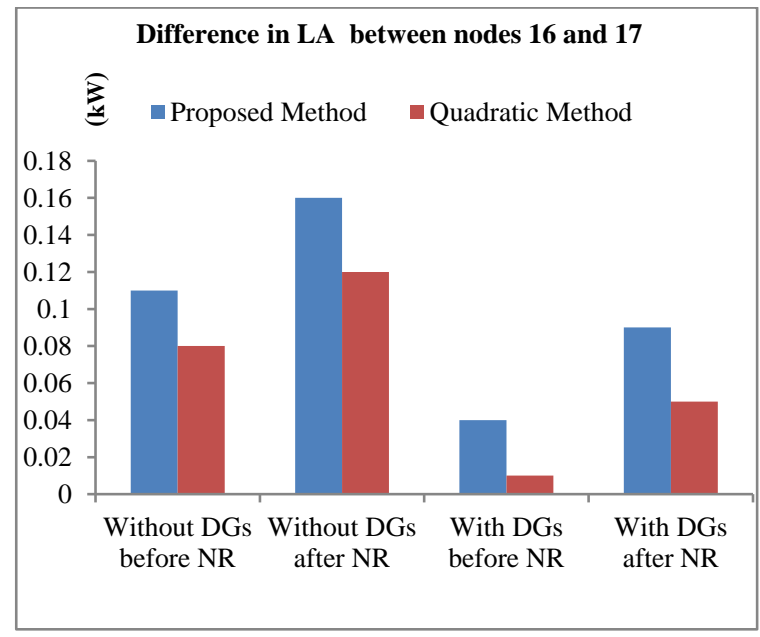

Figure 7. Difference in LA between nodes 16 and 17

\section{CONCLUSION}

The impact of NR on system loss allocation has been analysed in this paper with a new LA technique. The proposed BE based NR technique is simple to understand and easy to implement. Again, the loss allocation method developed eliminates the effects of cross-term mathematically from the loss formulation without any assumptions and approximations. The effectiveness of the proposed LA technique is verified by comparing the LA results with the established "Quadratic Method" with/without DGs at both situations of the network, i.e. before and after NR. The results are found to be fair and promising. It allocates losses to the network participants with due consideration to their load demands and geographical locations. Simultaneously, it also distributes the benefit of NR and DG power injection among the network participants, judiciously.

\section{REFERENCES}

[1] A. G. Exposito, J. R. Santos, T. G. Garcia, and E. R. Velasco, "Fair Allocation of Transmission Power Losses," IEEE Transactions on Power Systems, vol. 15, no. 1, pp. 184-188, 2000.

[2] S. Mishra, D. Das, and S. Paul, "A Simple Algorithm for Distribution System Load Flow with Distributed Generation," In Int. Conf. on Recent Advan. and Innovations in Engineering, pp. 1-5, 2014.

[3] A. J. Conejo, J. M. Arroyo, N. Alguacil, and A. L. Guijarro, "Transmission Loss Allocation: A Comparison of Different Practical Algorithms," IEEE Transactions on Power Systems, vol. 17, no. 3, pp. 571-576, 2002.

[4] E. M. Baran, and F. F. Wu, "Network Reconfiguration in Distribution Systems for Loss Reduction and Load Balancing," IEEE Trans Power Del., vol. 4, no. 2, pp. 1401-7, 1989.

[5] M. Atanasovski, and R Taleski, "Power Summation Method for Loss Allocation in Radial Distribution Networks with DG," IEEE Transactions on Power Systems, vol. 26, no. 4, pp. 2491-2499, 2011.

[6] M. Atanasovski, and R Taleski, "Energy Summation Method for Loss Allocation in Radial Distribution Networks with DG," IEEE Transactions on Power Systems, vol. 27, no. 3, pp. 1433-1440, 2012.

[7] Z. Ghofrani-Jahromi, Z. Mahmoodzadeh, and M. Ehsan, "Distribution Loss Allocation for Radial Systems including DGs," IEEE Transactions on Power Delivery, vol. 29, no.1, pp. 72-80, 2014.

[8] K. M. Jagtap, and D. K. Khatod, "Loss Allocation in Radial Distribution Networks with Different Load Models and Distributed Generations," IET Generation, Transmission \& Distribution, vol. 9, no. 12, pp. 1275-1291, 2015.

[9] S. S. Kashyap, and M. De "A Novel Loss Allocation Method for Radial Distribution System with Distributed Generations," 1st IEEE Int. Conf. on Power Electronics. Intelligent Control and Energy Systems, pp. 1-6, 2016.

[10] S. Ghaemi, and K. Zare, "Loss Allocation in Restructured Radial Distribution Networks Considering the Contractual Power," IET Generation, Transmission \& Distribution, vol. 11, no. 6, pp. 1389-1397, 2016.

[11] K. M. Jagtap, and D. K. Khatod, "Novel Approach for Loss Allocation of Distribution Networks with DGs," Electric Power System Research, vol. 143, pp. 303-311, 2017.

[12] S. Sharma, and A. R. Abhyankar, "Loss Allocation for Weakly Meshed Distribution System using Analytical Formulation of Shapley Value,” IEEE Transactions on Power Systems, vol. 32, no. 2, pp. 1369-1377, 2017.

[13] P. Kumar, N. Gupta, K. R. Niazi, and A. Swarnkar, "A Circuit Theory-based Loss Allocation Method for Active Distribution Systems," IEEE Transactions on Smart Grid., vol. 10, no. 1, pp. 1005-12, 2019.

[14] S. Mishra, D. Das, and S. Paul, "A Comprehensive Survey on Power Distribution Network Reconfiguration," Energy Systems, vol. 8, no. 2, pp. 227-284, 2017. 
[15] M. A. Muhammad, et al., "Integrated Database Approach in Multi Objective Network Reconfiguration for Distribution System using Discrete Optimisation Techniques," IET Generation Transmission Distribution, vol. 12, no. 4, pp. 976-986, 2018.

[16] Y. Ch, S. K. Goswami, and D. Chatterjee, "Effect of Network Reconfiguration on Power Quality of Distribution System," International Journal of Electrical Power \& Energy Systems, vol. 83, pp. 87-95, 2016.

[17] S. Ghasemi, "Balanced and Unbalanced Distribution Networks Reconfiguration Considering Reliability Indices," in Shams Engineering Journal, vol. 9, no. 4, pp. 1567-1579, 2016.

[18] S. R. Gampa, and D. Das, "Multi-Objective Approach for Reconfiguration of Distribution Systems with Distributed Generations," Electric Power Components and Systems, vol. 45, no. 15, pp. 1678-1690, 2017.

[19] S. Das, D. Das, and A. Patra, "Reconfiguration of Distribution Networks with Optimal Placement of Distributed Generations in the Presence of Remote Voltage Controlled Bus," Renewable and Sustainable Energy Reviews, vol. 73, pp. 772-781, 2017.

[20] G. Gutiérrez-Alcaraz, and J. H. Tovar-Hernández, "Two-Stage Heuristic Methodology for Optimal Reconfiguration and Volt/VAr Control in the Operation of Electrical Distribution Systems," IET Generation Transmission Distribution, vol. 11, no. 16, pp. 3946-3954, 2017.

[21] A. Tyagi, A. Verma, and R. P. Bijwe, "Reconfiguration for Loadability Limit Enhancement of Distribution Systems," IET Generation Transmission Distribution, vol. 12, no. 1, pp. 88-93, 2018.

[22] K. Jasthi, and D. Das, "Simultaneous Distribution System Reconfiguration and DG sizing Algorithm Without Load Flow Solution," IET Generation Transmission Distribution, vol. 12, no. 6, pp. 1303-1313, 2018.

[23] M. E. Oliveira, et al., "Network Reconfiguration and Loss Allocation in a Deregulated Environment of Distribution Systems," In Proc. of the 18th Int. Conf. on Electricity Distribution, pp. 6-9, 2005.

[24] A. J. Conejo, F. D. Galiana, and I. Kockar, "Z-Bus Loss Allocation," IEEE Transactions on Power Systems, vol. 16, no. 1, pp. 105-110, 2001.

[25] J. S. Savier, and D. Das, "Impact of Network Reconfiguration on Loss Allocation of Radial Distribution Systems," IEEE Transactions on Power Delivery, vol. 22, no. 4, pp. 2473-2480, 2007.

[26] C. Djabali, A. Boukaroura, N. Ketfi, and T. Bouktir, "Optimum Distribution Network Reconfiguration using Firefly Algorithm," Proc. of the Int. Conf. on Recent Advances in Electrical Systems, pp. 287-283, 2016.

[27] M. Quintero-Duran, E. J. Candelo, and J. Soto-Ortiz, "A Modified Backward/Forward Sweep-based Method for Reconfiguration of Unbalanced Distribution Networks," International Journal of Electrical and Computer Engineering (IJECE), vol. 9, no. 1, pp. 85-101, 2019.

[28] S. A. Reddy, D.M. Reddy, and K. S. M. Reddy, "Network Reconfiguration of Primary Distribution System Using GWO Algorithm," International Journal of Electrical and Computer Engineering (IJECE), vol. 7, no. 6, pp. 3226-3234, 2017.

[29] S. R. Rao, et al., "Power Loss Minimization in Distribution System using Network Reconfiguration in the Presence of Distributed Generation," IEEE Transactions on Power Systems, vol. 28, no. 1, pp. 317-325, 2013.

\section{BIOGRAPHIES OF AUTHORS}

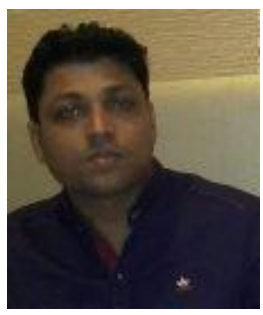

Ambika Prasad Hota received his B.E degree in Electrical Engineering in 2005 from Biju Patnaik University of Technology, India, and his M.Tech degree in Electrical Engineering in 2013 from Indian Institute of Technology, Kharagpur, India. He is currently pursuing his $\mathrm{PhD}$ program in International Institute of Information Technology, Bhubaneswar, India in Electrical Engineering with emphasis in Smart Grid management systems. His research interests include operation and control of power systems, renewable energies, and network modelling

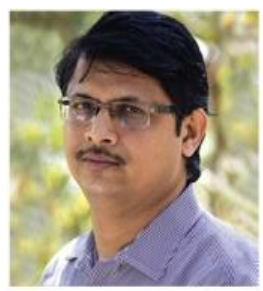

Sivkumar Mishra received his B.E degree in Electrical Engineering from Malaviya Regional Engineering College, Jaipur affiliated to University of Rajastan in 1995, M.Tech degree from Indian Institute of Technology, Kharagpur, India, and his PhD degree from Jadavpur University, India. He is currently working as Associate Professor in the department of Electrical Engineering, CAPGS, BPUT, Rourkele, India. His research interests include Electrical Power distribution system analysis, Distributed Generation and Micro Grid, Smart Grid, Application of IOT \& Big data analytics in Electrical power systems. 